NOTICE: this is the author's version of a work that was accepted for publication in Carbon. Changes resulting from the publishing process, such as peer review, editing, corrections, structural formatting, and other quality control mechanisms may not be reflected in this document. Changes may have been made to this work since it was submitted for publication. A definitive version was subsequently published in Carbon, Vol. 54 (2013).

DOI: 10.1016/j.carbon.2012.11.014 


\title{
Fullerene attachment to sharp-angle nanocones mediated by covalent oxygen bridging
}

\author{
I. Suarez-Martinez ${ }^{1,2}$, J. Mittal ${ }^{3}$, H.Allouche ${ }^{3}$, M. Pacheco ${ }^{4}$, \\ M. Monthioux ${ }^{3}$, M. Razafinimanana ${ }^{4}$, C.. P. Ewels ${ }^{1^{*}}$ \\ ${ }^{1}$ Institut des Matériaux Jean Rouxel, UMR6502 CNRS, University of Nantes, 2 rue de la Houssinière, \\ F-44322 Nantes, France \\ ${ }^{2}$ Nanochemistry Research Institute, Department of Chemistry, Curtin University of Technology, P.O. \\ Box U1987, Perth, WA, 6845, Australia \\ ${ }^{3}$ Centre d'Elaboration des Matériaux et d'Etudes Structurales, UPR-8011 CNRS, University of \\ Toulouse, BP 94347, F-31055 Toulouse, Cedex 4, France \\ ${ }^{4}$ LAPLACE, UMR 5002 CNRS, University of Toulouse, 118 Route de Narbonne, F-31062 Toulouse \\ cedex 4, France
}

\begin{abstract}
Using a combination of transmission electron microscopy and density functional modeling we examine covalent bridging between carbon nanoforms, focusing on fullerene attachment to carbon nanocones (nanohorns). We show that oxygen mediates covalent cross-linking between carbon nanoforms, analogously to oxygen-mediated fullerene dimerisation $\left(\mathrm{C}_{120} \mathrm{O}\right)$. We confirm this theoretically and experimentally for fullerenes bonded to nanocone tips. Oxygen bridging only occurs in systems with relatively localized double bond character, i.e. bridging only occurs between fullerenes and high angle nanocone tips.
\end{abstract}

* Corresponding author: E-mail address: chris.ewels@cnrs-imn.fr (C. P. Ewels)

\section{Introduction}


Single oxygen atom addition was the first functionalisation observed in fullerene chemistry [1]. The addition of oxygen to carbon-carbon bonds in $\mathrm{C}_{60}$ molecules can result in two possible structure as depicted in Figure 1a and b. Addition to pentagon-hexagon ([pent:hex]) bonds which primarily single character results in annulene forms (ether oxa-homo[60]fullerene), by breaking the C-C bond (Figure 1a). In the case of pentagon-hexagon ([pent:hex]) bond with a double bond character, oxidation results in a cycle-epoxide (Figure 1b) where the underlying $\mathrm{C}-\mathrm{C}$ bond beneath the oxygen is slightly dilated. The epoxide form can easily be obtained by photo-oxidation of $\mathrm{C}_{60}$ [2] or the reaction of $\mathrm{C}_{60}$ with dimethyldioxirane [3], however the annulene form requires photolysis of $\mathrm{C}_{60} \mathrm{O}_{3}$ prepared by ozonating $\mathrm{C}_{60}$ [4]. Oxygen is known to bridge between two $\mathrm{C}_{60}$ molecules through a furan-like ring forming $\mathrm{C}_{120} \mathrm{O}$ [5] and more recently between larger fullerenes [6]. This dimerisation reaction is based on the formation of a stable epoxide, which then reacts with a double bond on the neighbouring fullerene, cross-linking as shown in Figure 1c.

Carbon nanocones (also referred to as carbon nanohorns [7]) are commonly synthesized via laser ablation [8]. They can be described as $n \times 60^{\circ}$ positive wedge disclinations in graphene, and can be visualied by removing $n 60^{\circ}$ sections from graphene and connecting the exposed edges, forming $n$ pentagons at the apex. The disclination angle $(\alpha)$ can be calculated as $\alpha=2 \sin ^{-1}(1-n / 6)$, and since the number of pentagons must be an integer, nanocones only have tip angles of $113^{\circ}, 84^{\circ}, 60^{\circ}, 39^{\circ}$ and $19^{\circ}$, for $n=1,5$ respectively. The precise location of the pentagons can be varied [9]. Oxidative nanocone etching has received attention since it provides chemical access to the interior cone cavity $[10,11,12]$. However little is known of early stage and mild oxidation behaviour of carbon nanocones, and their low structural symmetry means they have been difficult to study theoretically.

We have previously examined direct fullerene-nanocone interaction via density functional calculations [13]. These show that while weak-covalent and dispersion forces are sufficient to bind $\mathrm{C}_{60}$ to carbon nanocones, there is negligible spatial or orientation dependence in the interaction, and no clear trend in binding with cone-tip angle. Notably the calculations give stronger binding of $\mathrm{C}_{60}$ to flat graphene 
surfaces than the exterior of cone tips, suggesting that in the absence of other factors fullerenes should migrate away from nanocone tips.

Here we examine fullerene-nanocone interaction mediated through oxygen bridging, both experimentally through electron microscopy imaging, and via density functional calculations.

\section{Method}

We perform spin polarized Density Functional calculations [14,15] within the Local Density Approximation, which has been shown to give $\mathrm{O}_{2}$ binding energy to graphene as well as $\mathrm{O}_{2}$ singlet/triplet energy differences closer to experiment than the generalized gradient approximation [16]. Ab initio calculations on nanocones are computationally expensive as they require atomic models with a large number of atoms and low symmetry. We study here an $84^{\circ}$ cone with two pentagons at the tip separated by a $\mathrm{C}-\mathrm{C}$ bond in a model of $274 \mathrm{C}$ atoms and $34 \mathrm{H}$ to saturate the edge $\left(\mathrm{C}_{274} \mathrm{H}_{34}\right)$, shown in Figure $2 \mathrm{a}$, as well as an $113^{\circ}$ cone with a single pentagon $\left(\mathrm{C}_{95} \mathrm{H}_{25}\right)$. Supercells were large enough to avoid interaction between molecules in neighboring cells. Structures were geometrically optimized with a single $k$-point. Hartwigsen, Goedecker and Hütter relativistic pseudopotentials were used for all atoms [17]. Atom-centered Gaussian basis functions were used to construct the many-electron wave function with angular momenta up to $l=2$ (22 independent functions per $\mathrm{C}$ atom, 40 per $\mathrm{O}$ ). Electronic level occupation was obtained using a Fermi occupation function with $k T=0.04 \mathrm{eV}$.

In the energetic analysis that follows, reaction enthalpies are calculated as the energy difference between the oxidized carbon nanoform and the sum of energy of the non-oxidized form and the $\mathrm{O}_{2}$ molecule in the stable triplet state, i.e. $\mathrm{E}_{\mathrm{R}}=\mathrm{E}\left(\right.$ Form-O) $-\mathrm{E}($ Form $)-1 / 2 \mathrm{E}\left(\mathrm{O}_{2}\right)$. Thus $\mathrm{E}_{\mathrm{R}}$ provides a measure for the exothermicity of oxidation.

Nanocone and fullerene production was carried-out using a vertical electric-arc arrangement. Standard arc parameters were used (80A current intensity, $\sim 30 \mathrm{~V}$, He atmosphere, 60-65 kPa reactor pressure, 1 $\mathrm{mm}$ electrode distance). Graphite anodes with homogeneously dispersed catalyst $(0.6 \mathrm{wt} \% \mathrm{Ni}+0.6 \mathrm{wt} \%$ Co) were prepared by a pressureless sintering technique [18], resulting in Ni-Co alloy particles whose 
initial sizes in the anode ranged from 30 to $200 \mathrm{~nm}$. All the solid products formed in the four main sampling areas of the reactor (i.e., so-called cathode deposit, collaret, web, and soot) were investigated "as-produced" upon sonicated dispersion in ethanol by high resolution transmission electron microscopy (HRTEM, Philips EM400, at $120 \mathrm{kV}$ with tungsten source, or Philips CM30, at $150 \mathrm{kV}$ with $\mathrm{LaB}_{6}$ source). The primary reaction product was carbon nanohorns, along with lesser amounts of spoorly organized polyaromatics, onion-like particles and fullerenes.

\section{Results and discussion}

We examine possible binding sites for oxygen on a carbon nanocone with two pentagons at the tip $\left(84^{\circ}\right.$ cone). In separate calculations we geometrically optimized the addition of oxygen to four different bonds, labeled 1-4 in Figure 2b. Bond \#1 lies at the tip of the cone, while bonds \#2-4 lie on the cone sidewalls. Bond \#1 is very similar to a [hex:hex] bond in $\mathrm{C}_{60}$ connecting the vertices of two pentagons. Bonds \#2-4 are further away from the cone tip. Bond \#2 lies mostly axial to the tip of the cone while bonds \#3 and \#4 are mostly circumferential.

The oxidation enthalpies and resultant $\mathrm{C}-\mathrm{C}$ and $\mathrm{C}-\mathrm{O}$ bond lengths are given in Table 1, along with values for equivalent oxidation of pristine graphene, and hex-hex and hex-pent bonds in $\mathrm{C}_{60}$ for comparison. Oxidation of bond $\# 1$ in the nanocone is exothermic $(-0.84 \mathrm{eV})$, resulting in an epoxide (underlying C-C bond is dilated to $1.48 \AA$ with $\mathrm{C}-\mathrm{O}$ bond lengths of $1.43 \AA$ ). The structure and formation energy resemble the oxidation of the [hex:hex] bond in $\mathrm{C}_{60}$, demonstrating the similarity of the nanocone tip to fullerene chemistry. On the nanocone sidewalls, bonds \#2 and \#3 give endothermic epoxides, while bond \#4 gives an exothermic annulene form. The 'extreme' case of oxidation of a flat graphene is an endothermic epoxide with formation energy $+0.59 \mathrm{eV}$.

Preferentially oxidized bonds will be those resulting in epoxide bonding with highest binding energies for oxygen. Thus for the $84^{\circ}$ nanocone this suggests preferential oxygen attachment at the double-bond like $\mathrm{C}-\mathrm{C}$ bond at the nanocone tip. 


\begin{tabular}{|c|c|c|c|c|}
\hline Nanoform & $\begin{array}{l}\text { Oxidation } \\
\text { reaction enthalpy } \\
(\mathrm{eV})\end{array}$ & $\begin{array}{l}\text { Underlying C-C } \\
\text { distance }(\AA)\end{array}$ & $\begin{array}{l}\text { Epoxide / } \\
\text { Annulene }\end{array}$ & $\begin{array}{l}\text { Oxidised bond } \\
\text { location }\end{array}$ \\
\hline $\mathrm{C}_{60}$ & $\begin{array}{l}-1.17 \\
-1.16\end{array}$ & $\begin{array}{l}1.53 \\
2.13\end{array}$ & $\begin{array}{l}\text { Epoxide } \\
\text { Annulene }\end{array}$ & $\begin{array}{l}\text { Hex:Hex } \\
\text { Pent:Hex }\end{array}$ \\
\hline $84^{\circ}$ Nanocone & $\begin{array}{l}-0.84 \\
+0.47 \\
+0.03 \\
-0.56\end{array}$ & $\begin{array}{l}1.48 \\
1.48 \\
1.59 \\
2.09\end{array}$ & $\begin{array}{l}\text { Epoxide } \\
\text { Epoxide } \\
\text { Epoxide } \\
\text { Annulene }\end{array}$ & $\begin{array}{l}\text { \#1 Tip Hex:Hex } \\
\# 2 \\
\# 3 \\
\# 4\end{array}$ \\
\hline Graphene & +0.59 & 1.514 & Epoxide & \\
\hline
\end{tabular}

Table 1 : Reaction enthalpy and resultant $\mathrm{C}-\mathrm{C}$ distance for addition of a single oxygen atom to $\mathrm{C}_{60}$, graphene and a $84^{\circ}$ two-pentagon carbon nanocone (structure and bond numbers in Figure $2 \mathrm{~b}$ ).

The results suggest the potential for the presence of oxygen at "fullerene-like" nanocone tips, but not on pristine sidewalls. Furthermore, oxidation will only be likely at sharper nanocone tips with multiple pentagons in reasonably close proximity. It is difficult to directly verify such a prediction experimentally since oxygen concentration at nanocone tips will be extremely low, and highly spatially resolved techniques would be required to avoid detecting oxygen elsewhere in the sample. However indirect validation is possible by considering possible reactions involving oxygenated sites as we now show. If we predict epoxides at some nanocone tips, oxygen mediated covalent bonding may also be possible at these locations, which would provide a mechanism to stably attach fullerenes to the nanocone tips.

We have therefore next calculated the enthalpy of reaction between $\mathrm{C}_{60}$ and $X$ to give $\mathrm{C}_{60} X$, where $X$ is either $\mathrm{C}_{60} \mathrm{O}$, an oxidized nanocone tip or an epoxide on graphene. Figure 3 shows graphically the possible reactions. The reaction between $\mathrm{C}_{60}$ and the oxidised carbon nanoforms is exothermic in all these cases. However, in the case of fullerene-O-graphene the further dissociation into $\mathrm{C}_{60} \mathrm{O}$ and graphene is 
exothermic, suggesting that fullerenes will scavange the oxygen atom from the flat graphene surface. In the case of $\mathrm{C}_{60}-\mathrm{O}-\mathrm{C}_{60}$ and $\mathrm{C}_{60} \mathrm{O}$-nanocone the result is a covalent bonding between the two carbon nanobjects mediated via the bridging oxygen atom, shown in more detail in Figure 4.

These calculations give a complete picture of the interaction between $\mathrm{C}_{60}$ and these other nanoobjects, described in detail below. They suggest that fullerenes should attach to sharp angle nanocone tips, but only in the presence of oxygen, since previous studies have ruled out any preferential fullerenenanocone tip attachment due to Van der Waals interactions or charge accumulation on the tip of the nanocone under the microscope [13].

To determine the importance of cone angle we also calculated oxygen and $\mathrm{C}_{60}$ interaction with a single pentagon $113^{\circ}$ cone. In this case the binding energy of $\mathrm{C}_{60} \mathrm{O}$ to a bond neighbouring the pentagon is significantly reduced at only $0.61 \mathrm{eV}$ (as compared to $2.01 \mathrm{eV}$ for the 2-pentagon cone). Thus oxygen mediated $\mathrm{C}_{60}$ bonding to single pentagon tips at elevated temperatures will be extremely unlikely. Furthermore higher angle cones where the pentagons are spatially separated will show local reactivites comparable to the single pentagon case, and hence it will only be in cones with clustered pentagons that we would expect oxygen mediated covalent bridging to occur. In practise this also eliminates 2-pentagon cones since there are so few configurations for which pentagons are in sufficiently close proximity.

We next used HRTEM to examine nanocone materials prepared by the electric arc method instead of the usual laser ablation. The electric arc method used with catalyst-loaded graphite anodes is known for concomitantly producing fullerenes and other single-walled structures such as single-wall carbon nanotubes. Due to the catalyst preparation conditions used the prevalent graphene-based forms were nanocones instead of single-wall nanotubes, in addition to fullerenes. In addition, while the reactor atmosphere is a partial pressure of helium, there is some oxygen present due to the large reactor volume, poor vacuum quality and the presence of large, highly absorbing metal parts. All the ingredients needed are thus present, resulting in fullerene-nanocone interaction features for which Figure 5 provides typical examples. As predicted by the calculations, we commonly observed fullerene-like molecules attached in 
the vicinity of the tip for nanocones with tip angles equal to $60^{\circ}$ or smaller i.e. 3 or more pentagons; however we never observed such attachment on any of the wider angle cones. This therefore confirms the presence of oxygen at the nanocone tips, mediating covalent oxygen bridging to the attached fullerene.

\section{Conclusions}

The calculations presented here allow a complete description of oxygen-mediated fullerene interaction with carbon nanocones. Both $\mathrm{C}_{60}$ and $\mathrm{C}_{60} \mathrm{O}$ will weakly physisorb on graphene and nanocone side walls, and hence these surfaces can act as capture areas. Any oxygen present on these surfaces will be scavenged by the $\mathrm{C}_{60}$ since this process is highly exothermic, but with no covalent bonds forming between the fullerene and the side walls, the fullerene will remain highly mobile. On migrating to the nanocone tip, assuming the presence of oxygen either on the fullerene cage or at the nanocone tip, covalent crosslinking will occur, stabilizing the fullerene in place. This pinning will only occur on cones with tip angles sufficiently small that there are multiple nearby pentagons and so high local curvature and localised double bond character. We note that similar pinning and cross-linking behaviour might be expected at point defect sites such as oxygenated vacancies on nanotube sidewalls, and this may provide an alternative model for some "nanobud" structures observed in the literature [19].

In summary, we show that oxygen can form covalent linking bridges between different carbon nanoforms directly analogous to fullerene dimers, for example between fullerenes and nanocone tips. We expect this to be a general phenomenon wherever oxidized $\mathrm{C}-\mathrm{C}$ bonds are present, for example on basal dislocations in nanotubes or at point defect sites such as vacancies.

\section{Acknowledgement}

IS acknowledges financial support from the Australian Research Council under the grant DP110104415. CE acknowledges the SPRINT project $n^{\circ}$ ANR-2010-BLAN-0819-04 funded by the French National Agency (ANR). 
1. Diederich F, Ettl R, Rubin Y, Whetten RL, Beck R, Alvarez M, et al. The higher fullerenes: isolation and characterization of $\mathrm{C}_{76}, \mathrm{C}_{84}, \mathrm{C}_{90}, \mathrm{C}_{94}$, and $\mathrm{C}_{70} \mathrm{O}$, an oxide of $D_{5 h}-\mathrm{C}_{70}$. Science 1991;252:548-51.

2. Creegan KM, Robbins J, Robbins W, Millar J, Sherwood R, Tindall P, et al.. Synthesis and characterisation of $\mathrm{C}_{60} \mathrm{O}$, the first fullerene epoxide. J Am Chem Soc 1992;114:1103-5.

3. Elemes Y, Silverman SK, Sheu C, Kao M, Foote CS, Alvarez M, et al. Reaction of $\mathrm{C}_{60}$ with dimethyldioxirane-Formation of an epoxide and a 1,3-dioxolane derivative. Angew Chem Int Ed Engl 1992;31:351-3.

4. Weisman RB, Heymann DM, Bachilo S. Synthesis and characterisation of the "missing" oxide of $\mathrm{C}_{60}$ : [5,6]-Open $\mathrm{C}_{60}$ O. J Am Chem Soc 2001;123:9720-1.

5. Smith-III AB, Tokuyama H, Strongin R, Furst G, Romanow W. Synthesis of oxo- and methylenebridged $\mathrm{C}_{60}$ dimers, the first well-characterised species containing fullerene-fullerene bonds.. $\mathrm{J}$ Am Chem Soc 1995;117:9359-60.

6. Weber L, Carta-Abelmann L, Ritter U, Scharff P. Preparation and characterization of the dimeric $\mathrm{C}_{70}$ fullerene oxide $\mathrm{C}_{140} \mathrm{O}$. Fuller, Nanotub Carb Nanostruct 2008;16:258-71.

7. Suarez-Martinez I, Grobert N, Ewels CP. Nomenclature of $\mathrm{sp}^{2}$ carbon nanoforms. Carbon 2012:50:741-7.

8. Iijima S, Yudasaka M, Yamada R, Bandow S, Suenaga K, Kokai F, et al. Nano-aggregates of singlewalled graphitic carbon nano-horns. Chem Phys Lett 1999;309:165-70.

9. Klein DJ. Topo-combinatoric categorization of quasi-local graphitic defects. Phys. Chem. Chem. Phys 2002;4:2099-110.

10. Utsumi S, Honda H, Hattori Y, Kanoh H, Takahashi K, Sakai H, et al. Direct evidence on C-C single bonding in single-wall carbon nanohorn aggregates. J Phys Chem C 2007;111:5572-5.

11. Yoshida S, Sano M. Microwave-assisted chemical modification of carbon nanohorns: Oxidation and Pt deposition. Chem Phys Lett 2006;433:97-100.

12. Pagona G, Tagmatarchis N, Fan J, Yudasaka M, Iijima S. Cone-end functionalisation of carbon nanohorns. Chem Mater 2006;18:3918-20.

13. Suarez-Martinez I, Monthioux M, Ewels CP. Fullerene interaction with carbon nanohorns. J Nanosci Nanotechnol 2009;9:6144-8.

14. Briddon PR, Jones R. LDA Calculations using a basis of Gaussian orbitals. Phys Stat Sol B 2000;217:131-71.

15. Rayson $\mathrm{MH}$, Briddon PR. Rapid iterative method for electronic-structure eigenproblems using localized basis functions. Comput Phys Commun 2008;178:128-34. 
16. Giannozzi P, Car R, Scoles G. Oxygen adsorption on graphite and nanotubes. J Chem Phys 2003;118:1003-7.

17. Hartwigsen C, Goedecker S, Hutter J. Relativistic separable dual-space Gaussian pseudopotentials from H to Rn. Phys Rev B 1998;58:3641-62.

18. Sogabe T, Matsuda T, Kuroda K, Hirohata Y, Hino T, Yamashina T. Preparation of $\mathrm{B}_{4} \mathrm{C}$-mixed graphite by pressureless sintering and its air oxidation behaviour. Carbon 1995;33:1783-8.

19. Nasibulin AG, Pikhitsa PV, Jiang H, Brown DP, Krasheninnikov AV, Anisimov AS, et al. A novel hybrid carbon material. Nature Nanotechnol 2007;2:156-61.

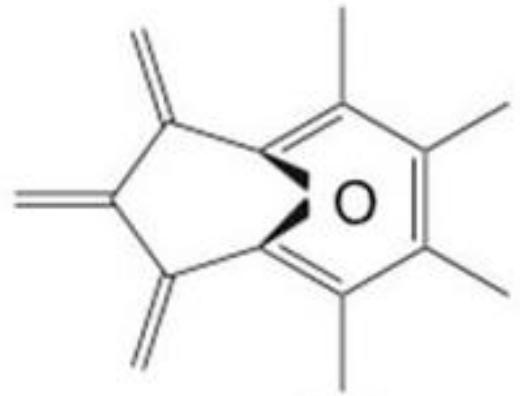

(a)

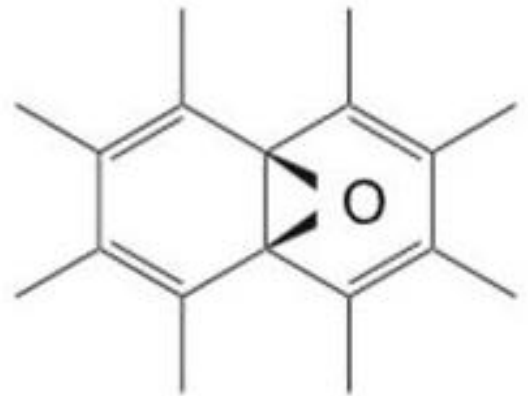

(b)
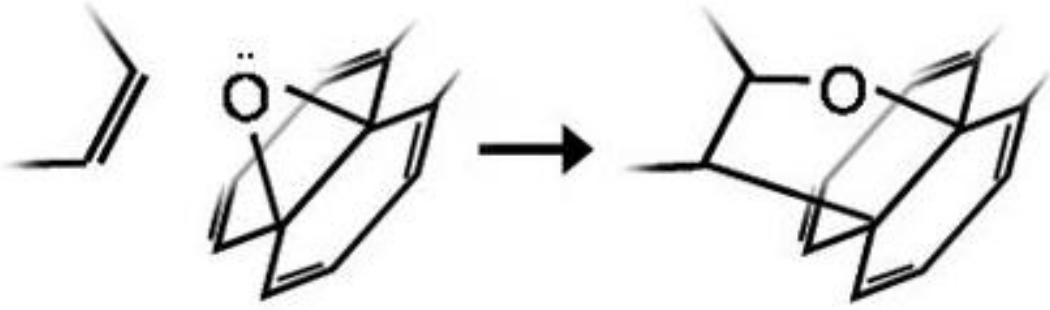

(c)

Figure Error! Main Document Only.. Isomers of an oxidized fullerene: a) 1,5-oxido[9]annulene on a [pent:hex] bond b) epoxide on a [hex:hex] bond. (c) Oxygen mediated 2+2 cycloaddition reaction, covalently linking two carbon nanoobjects via $\mathrm{C}-\mathrm{C}$ double bonds in proximity. 

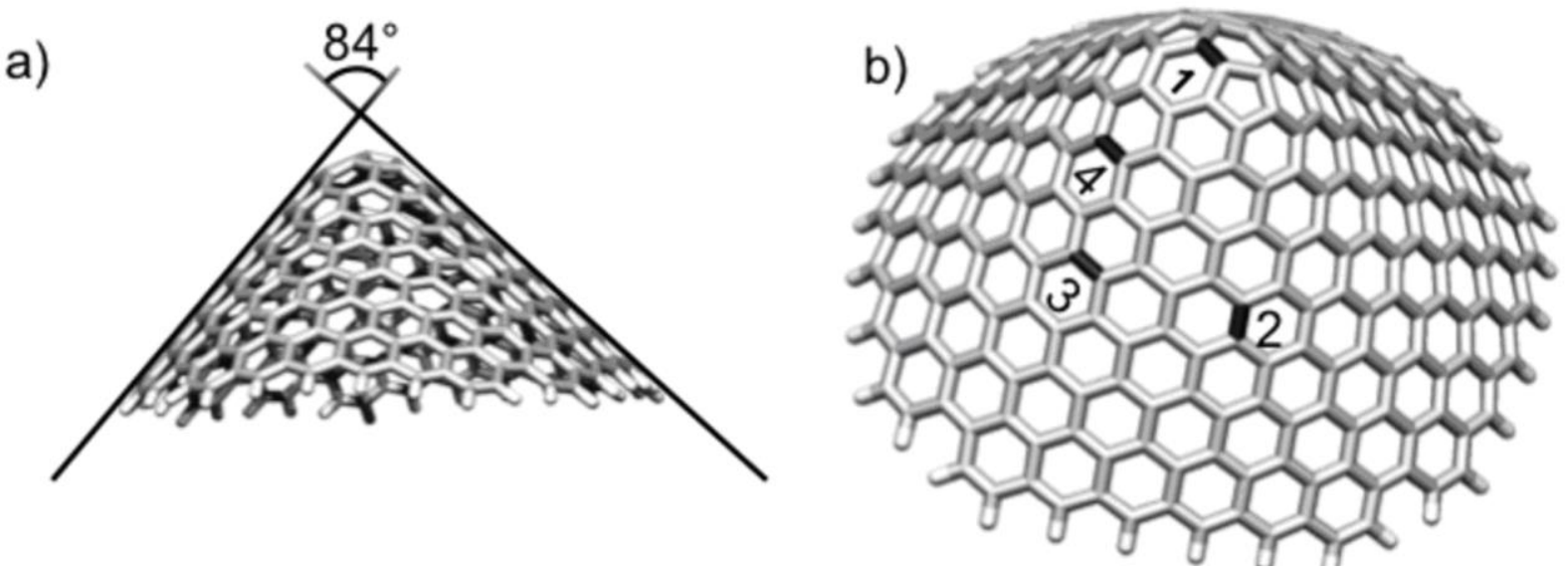

Figure 2. (a) Side view of an $84^{\circ}$ nanocone with two pentagons at the tip separated by a C-C bond. In (b) bonds which have been oxidized and geometrically optimized by DFT are marked in black.

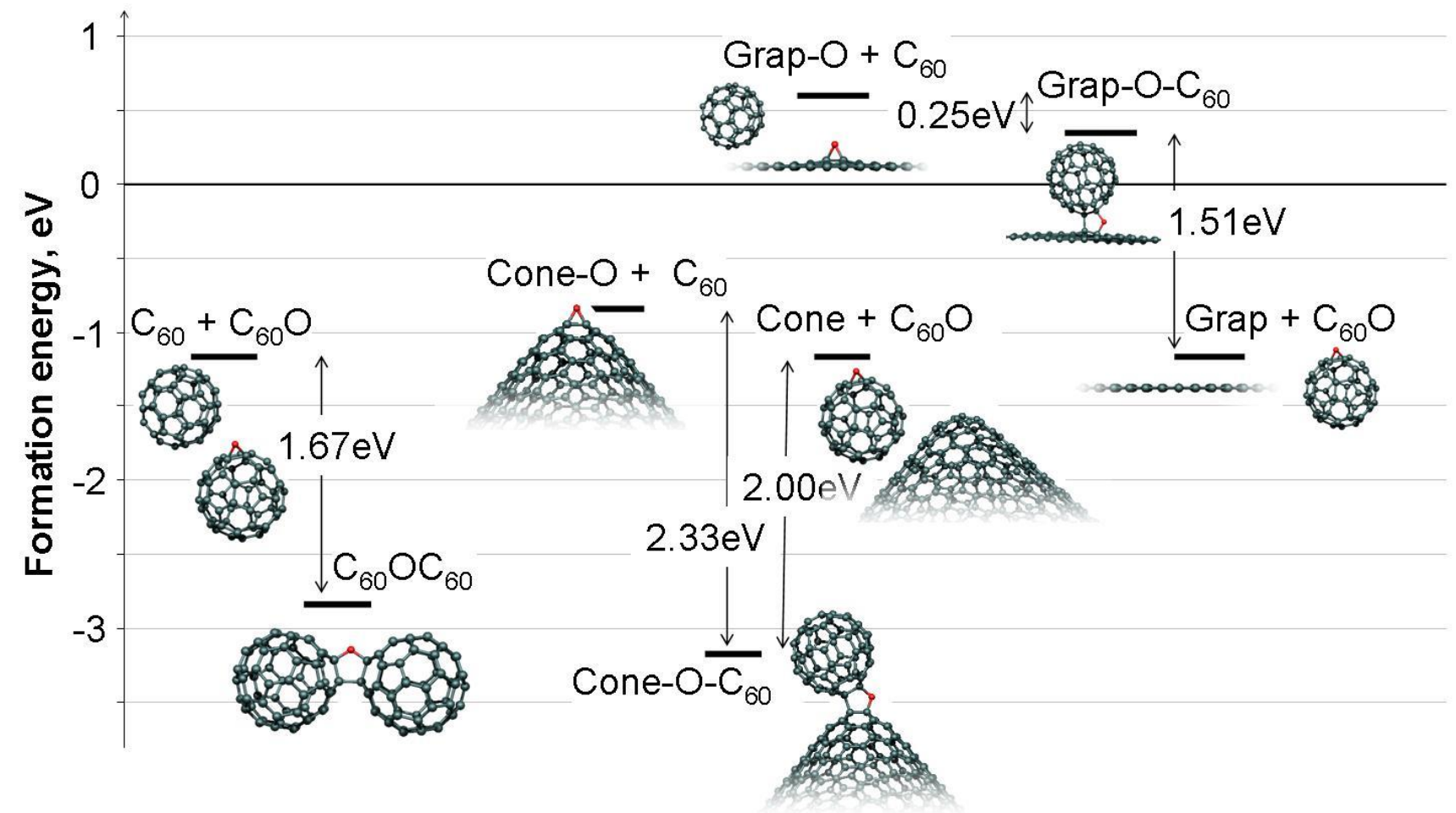

Figure 3. DFT calculated reaction enthalpies (in eV) for oxygen bridging between $\mathrm{C}_{60}$ and $X$, where $X$ is $\mathrm{C}_{60} \mathrm{O}$, oxidized $84^{\circ}$ nanocone tip or an epoxide on graphene. Energies are relative to isolated nonoxidized nanoforms and $1 / 2 \mathrm{O}_{2}$. 
(a)

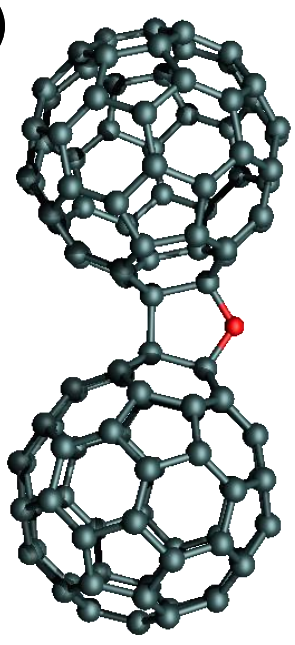

(b)

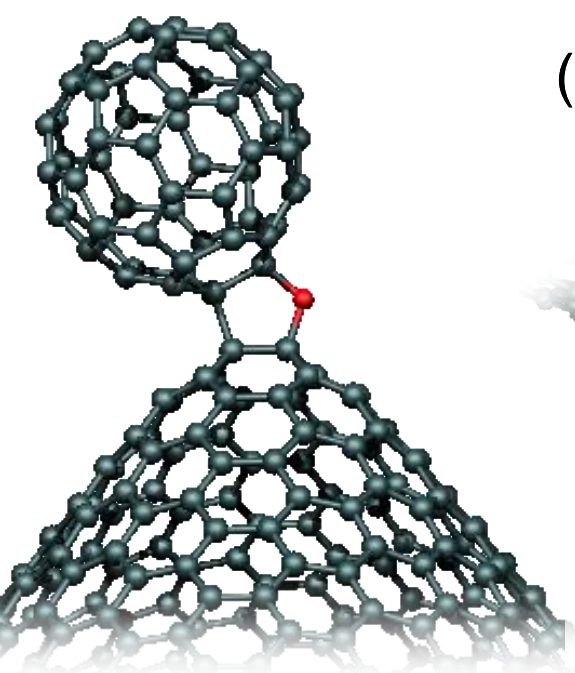

(c)

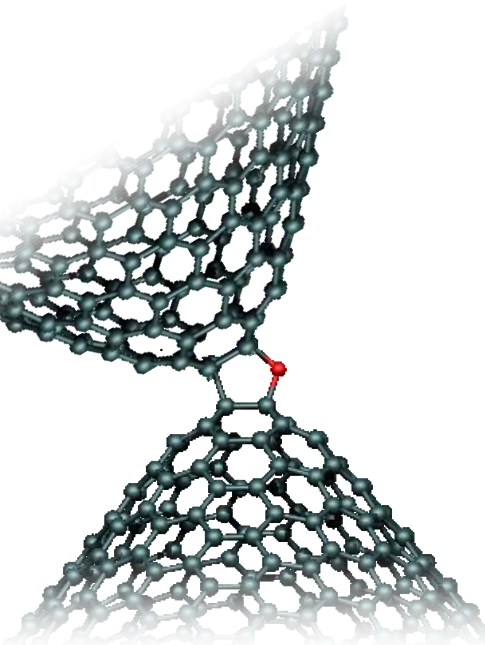

Figure 4 : DFT relaxed structures for (a) $\mathrm{C}_{60}-\mathrm{O}-\mathrm{C}_{60}$ and (b) $\mathrm{C}_{60}-\mathrm{O}-84^{\circ}$ nanocone showing oxygen mediated covalent cross-linking in both cases, (c) Hypothetical equivalent $84^{\circ}-\mathrm{O}-84^{\circ}$ bridging. 


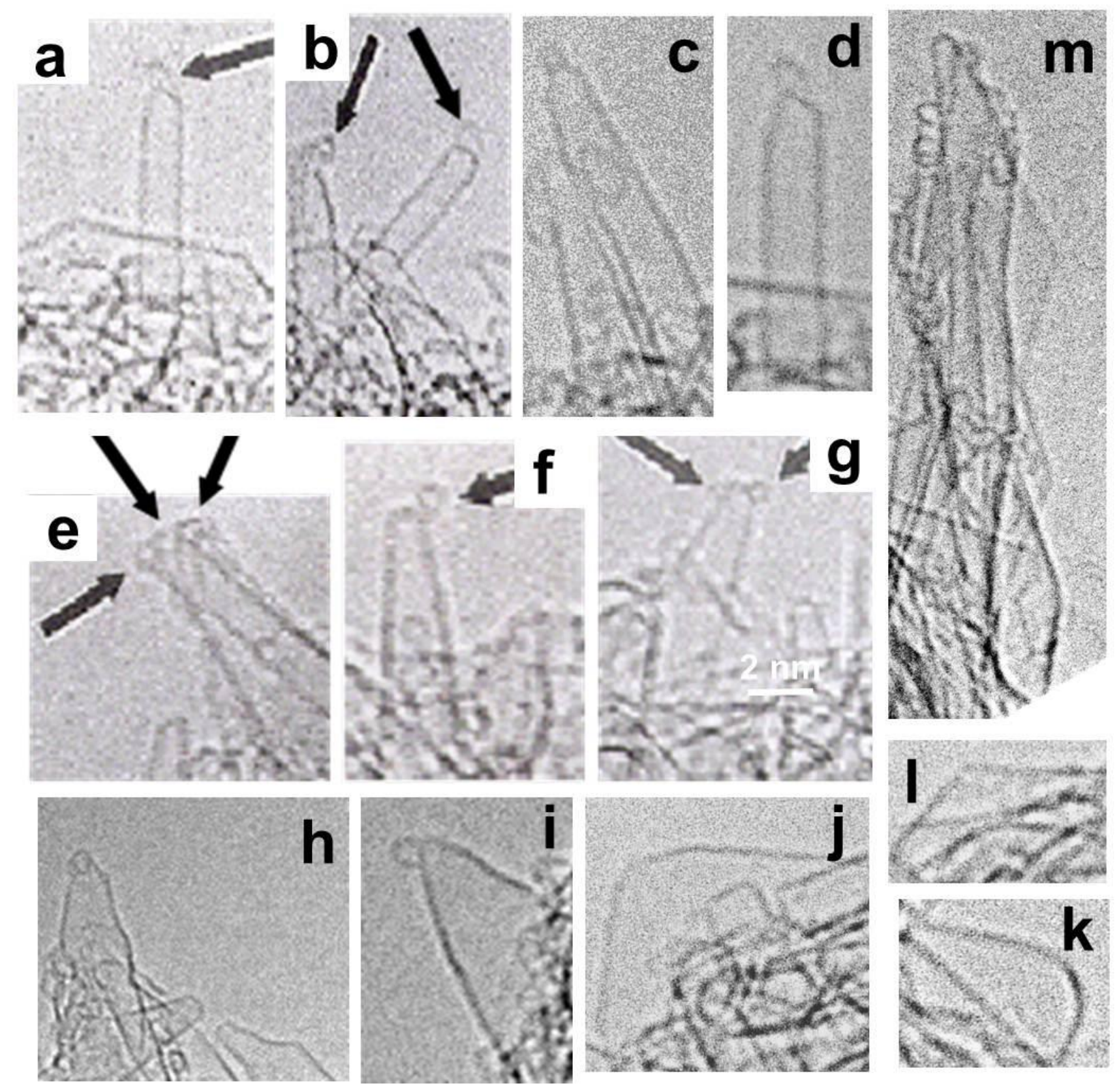

Figure 5. HRTEM showing examples of fullerene molecules adsorbed onto single wall nanocones in the vicinity of the nanocone tip for sharp angled tips only (from a) to i)) while there is no fullerene attached for large angles (from $\mathrm{j}$ ) to $\mathrm{m}$ )). The fullerenes involved are not necessarily all $\mathrm{C}_{60}$, but can be higher fullerenes. The cone angle value is not always equal to the ideal value because it may result from the projection of the tilted structure. a) to d) $0^{\circ}$ tip (6 pentagons). e) to $\mathrm{g}$ ) $\sim 19^{\circ}$ nanocones (5 pentagons); image e) actually shows a $0^{\circ}$ tip and a $19^{\circ}$ cone superimposed. h) and i) $\sim 30^{\circ}$ nanocones ( 4 pentagons). j) 
and $\mathrm{k}) \sim 84^{\circ}$ nanocones (3 pentagones). 1 ) and $\left.\mathrm{m}\right) \sim 113^{\circ}$ nanocones ( 1 pentagon); image $\mathrm{m}$ ) shows three aligned examples of it; other examples can be seen in a) and b) as well. 